\title{
Nanoscale Atomic Density Microscopy
}

\author{
S. Subhankar, Y. Wang, ${ }^{*}$ T-C. Tsui, S. L. Rolston, and J. V. Porto \\ Joint Quantum Institute, National Institute of Standards and Technology and the University of Maryland, \\ College Park, Maryland 20742 USA
}

(Received 10 October 2018; revised manuscript received 14 December 2018; published 1 April 2019)

\begin{abstract}
Quantum simulations with ultracold atoms typically create atomic wave functions with structures at optical length scales, where direct imaging suffers from the diffraction limit. In analogy to advances in optical microscopy for biological applications, we use a nonlinear atomic response to surpass the diffraction limit. Exploiting quantum interference, we demonstrate imaging with superresolution of $\lambda / 50$ and excellent temporal resolution of $500 \mathrm{~ns}$. We characterize our microscope's performance by measuring the ensemble-averaged probability density of atoms within the unit cells of an optical lattice and observe the dynamics of atoms excited into motion. This approach can be readily applied to image any atomic or molecular system, as long as it hosts a three-level system.
\end{abstract}

DOI: 10.1103/PhysRevX.9.021002

Subject Areas: Atomic and Molecular Physics, Optics, Quantum Physics

\section{INTRODUCTION}

High spatial and temporal resolution microscopy can reveal the underlying physics, chemistry, and biology of a variety of systems. Examples range from the study of atoms on surfaces with atomic resolution scanning tunneling microscopy [1] to the use of superresolution microscopy to observe individual molecule dynamics within living cells [2]. The field of quantum simulation with ultracold atoms has emerged to study strongly correlated many-body systems using precise control with light-atom interactions [3]. This entails confining atoms, engineering their interactions and potentials, and measuring their states with laser light. Based on fluorescence and absorption, the inherent imaging resolution is limited by diffraction. Bringing superresolution microscopy to the field of quantum simulation of condensed-matter systems with ultracold atoms will allow new direct probes of the wave function in a variety of many-body systems.

We demonstrate here the imaging of atoms with unprecedented spatial resolution approximately equal to $11 \mathrm{~nm}$ that is well below the diffraction limit. Our approach is based on a technique to localize atomic excitation on a subwavelength scale, first proposed by Agarwal and Kapale [4] and first demonstrated by Miles et al. [5]. We can directly measure the

\footnotetext{
* Corresponding author. wang.yang.phy@gmail.com

Published by the American Physical Society under the terms of the Creative Commons Attribution 4.0 International license. Further distribution of this work must maintain attribution to the author(s) and the published article's title, journal citation, and DOI.
}

atomic probability density optically within the unit cell of a 1D optical lattice (scanning electron microscopy has been used to measure such quantities with 150-nm resolution [6]), in contrast to measuring site occupancies $[3,7,8]$. Far-field microscopy at the nanoscale based on nonlinear optical response is well established [2] to resolve molecular dynamics inside biological samples. Using similar ideas, subwavelength addressing [9] and localized excitation have been proposed [10-16] and observed in atomic systems [5,17]. Based on the dark state associated with a three-level system $[5,9-14,16]$, we coherently shelve narrow slices of the wave function in every unit cell of the lattice into one of the spin states dictated by the local dark state. We selectively read out the total population in that spin state, which is proportional to the local probability density of the lattice wave function. The working resolution (width of the slice) can be adjusted by changing the dark-state composition. The coherent nature of this approach allows us to measure on a timescale much faster than the evolution of the wave function. Our setup can be readily applied to current quantum gas experiments [3]. By dispersively coupling the readout state to a cavity, as suggested by Refs. $[16,18]$, one could perform subwavelength quantum nondemolition measurements.

The principle of our approach is illustrated in Fig. 1 and is similar to Refs. $[4,5,16,18]$. Assuming adiabaticity, a threelevel atom [Fig. 1(a)] coupled by two spatially varying light fields will stay in a dark state, which is decoupled from the excited state $|e\rangle$. This dark state is a superposition of the two ground states with spatially varying amplitudes:

$$
|D(x)\rangle=\frac{1}{\sqrt{\Omega_{c}(x)^{2}+\Omega_{p}^{2}}}\left[\Omega_{c}(x)\left|g_{1}\right\rangle-\Omega_{p}\left|g_{2}\right\rangle\right] .
$$


(a)

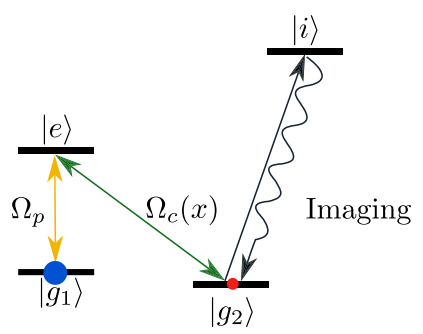

(b)

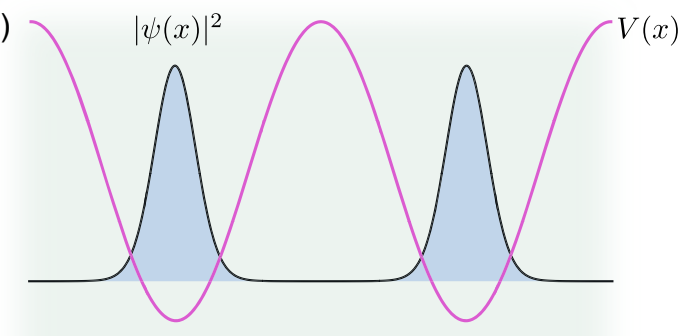

(c)

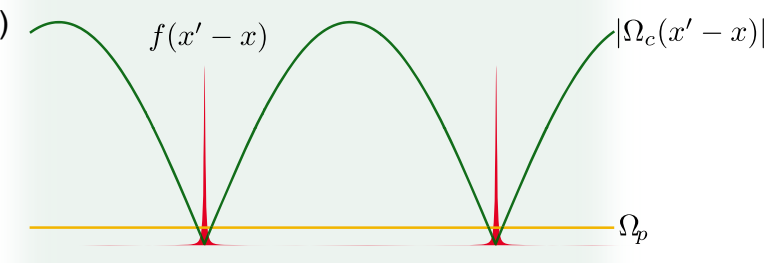

(d)

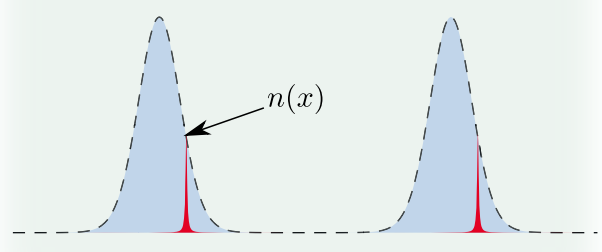

FIG. 1. Principle of our nanoscale atomic density microscope. (a) Configuration of the control field $\Omega_{c}(x)$ and probe field $\Omega_{p}$ coupling a $\Lambda$ system composed of $\left|g_{1}\right\rangle,\left|g_{2}\right\rangle$, and $|e\rangle$. Population in $\left|g_{2}\right\rangle$ is measured via a cycling transition connecting the imaging state $|i\rangle$. (b) Wave function density $|\psi(x)|^{2}$ in $\left|g_{1}\right\rangle$ in the lattice of interest $V(x)$. (c) The spin-state composition is transferred to $\left|g_{2}\right\rangle$ near the nodes of $\Omega_{c}\left(x^{\prime}-x\right)$ with probability given by $f\left(x^{\prime}-x\right)$ (narrow red peaks) and $\left|g_{1}\right\rangle$ elsewhere. The width of $f\left(x^{\prime}-x\right)$ is determined by the relative strength of the two light fields $\epsilon=$ $\Omega_{p} / \Omega_{c}$ [see Eq. (2)]. (d) $f\left(x^{\prime}-x\right)$ maps $|\psi(x)|^{2}$ onto the population in $\left|g_{2}\right\rangle, n(x)$, which can be selectively measured via state-dependent imaging. By stepping through different positions $x$ and measuring $n(x)$, we can reconstruct $|\psi(x)|^{2}$.

Here, we use a standing-wave control field $\Omega_{c}(x)=$ $\Omega_{c} \sin (k x)$ and homogeneous probe field $\Omega_{p}$, where $k=2 \pi / \lambda$, and $\lambda$ is the wavelength of the light. For $\Omega_{c} \gg \Omega_{p}$, the resulting dark-state composition is predominantly $\left|g_{1}\right\rangle$ away from the nodes of $\Omega_{c}(x)$, and $\left|g_{2}\right\rangle$ near the nodes where $\Omega_{p} \gg\left|\Omega_{c}(x)\right|$. The probability density of $\left|g_{2}\right\rangle$ [Fig. 1(c)] coming from this nonlinear dependence on the Rabi frequencies [Eq. (1)] is periodic and has narrow peaks near the nodes

$$
f(x)=\frac{\epsilon^{2}}{\epsilon^{2}+\sin ^{2}(k x)},
$$

where $\epsilon=\Omega_{p} / \Omega_{c}$. The full width at half maximum (FWHM) $\sigma$ of the peaks provides a good metric for the resolution within the unit cell $\lambda / 2$. For $\Omega_{c} \gg \Omega_{p}$ (small $\epsilon$ ), $\sigma$ depends linearly on $\epsilon: \sigma \simeq \epsilon \lambda / \pi$, allowing resolution greatly exceeding the diffraction limit. Starting with atoms in $\left|g_{1}\right\rangle$ with wave function $\psi(x)$, we can adiabatically transfer a narrow slice of atoms into $\left|g_{2}\right\rangle$. The wave-function probability density $|\psi(x)|^{2}$ [Fig. 1(b)] can be determined by measuring the population transferred to $\left|g_{2}\right\rangle$ at different locations $x$ [Fig. 1(d)], yielding a signal

$$
n(x)=\int\left|\psi\left(x^{\prime}\right)\right|^{2} f\left(x^{\prime}-x\right) d x^{\prime} .
$$

By deconvolving this signal with the probing function $f(x)$, we can reconstruct $|\psi(x)|^{2}$.

We use stimulated Raman adiabatic passage (STIRAP) [19] to transfer the selected slices of the wave function from the state $\left|g_{1}\right\rangle$ into $\left|g_{2}\right\rangle$. In order to accurately measure the shape of the wave function, the STIRAP process must be adiabatic with respect to the spin degree of freedom (d.o.f.) [i.e., the dark-state composition given by Eq. (1)] but diabatic with respect to the motional d.o.f. For small $\epsilon$, the shortest duration of the STIRAP is inversely proportional to the Rabi frequencies. For typical trapped atoms experiments, Rabi frequencies can be tens of megahertz, while the motional dynamics is on the order of tens of kilohertz.

\section{EXPERIMENTAL METHOD}

We work with the three-level system in ${ }^{171} \mathrm{Yb}$ consisting of $\left|g_{1}\right\rangle=\left|{ }^{1} S_{0}, F=\frac{1}{2}, m_{F}=-\frac{1}{2}\right\rangle,\left|g_{2}\right\rangle=\mid{ }^{1} S_{0}, F=\frac{1}{2}, m_{F}=$ $\left.+\frac{1}{2}\right\rangle$, and $|e\rangle=\left|{ }^{3} P_{1}, F=\frac{1}{2}, m_{F}=-\frac{1}{2}\right\rangle$ (see Appendix A), coupled by $\lambda=556 \mathrm{~nm}$ light. The control field $\Omega_{c}(x)$ is formed by two counterpropagating $\sigma^{-}$-polarized beams $\Omega_{c 1} e^{i k x}$ and $\Omega_{c 2} e^{-i k x}$ in the direction of the quantization axis defined by a magnetic field along $\hat{x}$, while the probe field $\Omega_{p}$ is a $\pi$-polarized traveling wave normal to the control beams [20]. We prepare ${ }^{171} \mathrm{Yb}$ atoms by sympathetically cooling them with ${ }^{87} \mathrm{Rb}$ atoms [21]. After ramping up the magnetic field to $36 \mathrm{mT}$ and removing the $\mathrm{Rb}$ atoms, the $\mathrm{Yb}$ atoms are optically pumped into $\left|g_{1}\right\rangle$ with a final population approximately equal to $2 \times 10^{5}$. We measure $|\psi(x)|^{2}$ of spin-polarized $\mathrm{Yb}$ atoms loaded into either a Kronig-Penney- (KP) type lattice of thin barriers, as described in Ref. [20], or a regular sinusoidal lattice based on the ac Stark shift of $\Omega_{c 1,2}$ off-resonantly coupled to the $\left|g_{1}\right\rangle \leftrightarrow\left|{ }^{3} P_{1}, F=\frac{3}{2}, m_{F}=-\frac{3}{2}\right\rangle$ transition, which lies outside the three-level system making up the dark state.

Our microscope is implemented as follows. We first suddenly turn off the lattice potential $V(x)$ that supports the wave function to be probed by switching off the $\Omega_{c 2}$ beam. 
Next, we ramp on $\Omega_{p}$ followed by $\Omega_{c 2}$ with a different phase, which adiabatically flips the spin from $\left|g_{1}\right\rangle$ to $\left|g_{2}\right\rangle$ in the region tightly localized near the nodes of the shifted $\Omega_{c}\left(x^{\prime}-x\right)=\Omega_{c} \sin \left[k\left(x^{\prime}-x\right)\right]$. The intensity profiles for ramping these two beams are calculated to preserve adiabaticity, ensuring atoms follow the spatiotemporal dark state at all times. We then rapidly ramp off all beams simultaneously in order to preserve the dark-state composition. We measure the $\left|g_{2}\right\rangle$ population via stateselective absorption imaging. Scanning $x$ in fine steps at small $\epsilon$ allows us to map out the $|\psi(x)|^{2}$ with high resolution.

\section{RESULTS}

We use our wave-function microscope to investigate atoms in sinusoidal and KP lattices. We start by preparing the atoms in the ground band of the lattice of interest, as described in Appendix B. Figure 2(a) shows $n(x)$ measured in a $140 E_{R}$ sinusoidal lattice using a calculated resolution of $8.8 \mathrm{~nm}$, along with $n(x)$ in a KP lattice with $50 E_{R}$ barriers using a calculated resolution of $17.6 \mathrm{~nm}$. Here, $E_{R}=\hbar^{2} k^{2} / 2 m$ is the recoil energy, and $m$ is the mass of the atom. The different lattice potentials (sinusoidal vs boxlike KP) give rise to different functional forms of the wave function in the lattice [inset of Fig. 2(a)]. The expected $|\psi(x)|^{2}$ is Gaussian for a deep sinusoidal lattice and cosine for a KP lattice. The solid lines are the calculated $|\psi(x)|^{2}$ using independently measured Rabi frequencies including both the resolution due to finite slice width as well as wave-function expansion over the 800-ns measurement time. In Fig. 2(b), we show the FWHM $w$ of the ground-band $|\psi(x)|^{2}$ of the sinusoidal lattice as a function of the lattice depth. The blue curve represents the calculated width, which is in good agreement with the data. The remaining discrepancy may result from trap inhomogeneities, the uncertainty of the Rabi frequencies, and mechanical effects arising from the nonadiabatic potentials due to the spatially varying dark state $[20,22,23]$.

The fast STIRAP slicing process allows for observing the wave-function dynamics. At our maximum Rabi frequency of $\Omega_{c}=2 \pi \times 90 \mathrm{MHz}$ and $\epsilon=0.05$, we can maintain the adiabaticity condition for a STIRAP time of $500 \mathrm{~ns}$. Figure 3(a) shows the dynamics of the wave function in a sinusoidal lattice after a sudden shift in the lattice position. The atoms are first adiabatically loaded into a $140 E_{R}$ lattice. Then, the lattice position is diabatically changed in $100 \mathrm{~ns}$ by $1 / 4$ of the lattice spacing via the phase of the $\Omega_{c 1}$ lattice beam, which excites a "sloshing" motion. We map out the evolution of the wave function within the unit cell by holding the atoms in the shifted lattice for incremental periods of time before probing. In Fig. 3(a), we show the measured $|\psi(x)|^{2}$, which are in agreement with the calculations.
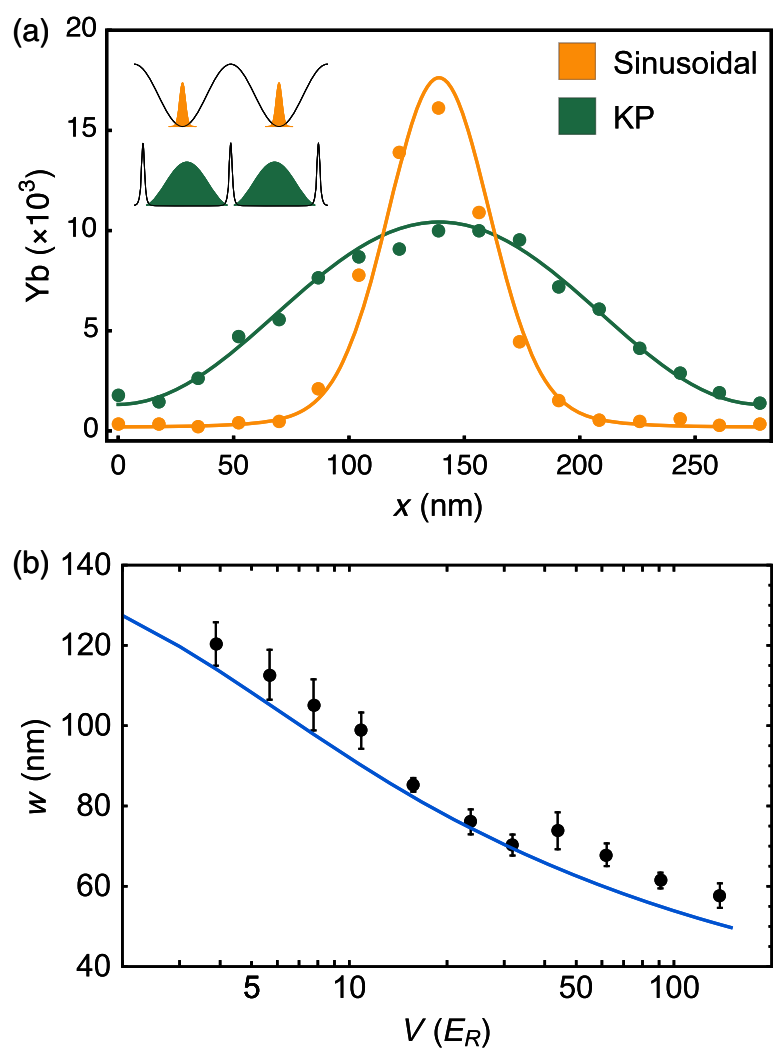

FIG. 2. Measurements of the ground-state wave function within the unit cell of an optical lattice with different shapes. (a) The orange points show $n(x)$ for atoms in a sinusoidal lattice measured with $\epsilon=0.05$. The green points represent $n(x)$ in a Kronig-Penney lattice measured with $\epsilon=0.1$. Number fluctuations between realizations result in number uncertainties of $5 \%$. The solid lines are calculations using measured Rabi frequencies to determine the lattice depth normalized to the same atom number. Inset: Schematic of different lattice potentials and corresponding $|\psi(x)|^{2}$. (b) FWHM $w$ of $n(x)$ in a sinusoidal lattice as a function of the lattice depth. Black points show experimental data with $\epsilon=0.05$, and the blue line is a calculation including the 800ns measurement time. The error bars are 1 standard deviation from the Gaussian fits.

The dynamics of the wave function after a sudden change in the lattice depth is shown in Fig. 3(b). The atoms are first adiabatically loaded into the ground band of a shallow lattice $\left(6 E_{R}\right)$. The depth of the lattice is then suddenly increased to $140 E_{R}$, which excites a "breathing" motion of the atoms inside a unit cell. As time increases, we see that the $|\psi(x)|^{2}$ breathes at a frequency characterized by the band energies. At $t=$ $3.5 \mu \mathrm{s}$ and $t=9.5 \mu \mathrm{s},|\psi(x)|^{2}$ is focused to calculated $w_{0} \simeq 26 \mathrm{~nm}$.

We estimate the spatial resolution of our microscope by measuring the narrowest wave function $|\psi(x)|^{2}$ that we create with the breathing-mode excitation. This condition occurs at $9.5 \mu$ s where the calculated $|\psi(x)|^{2}$ has 


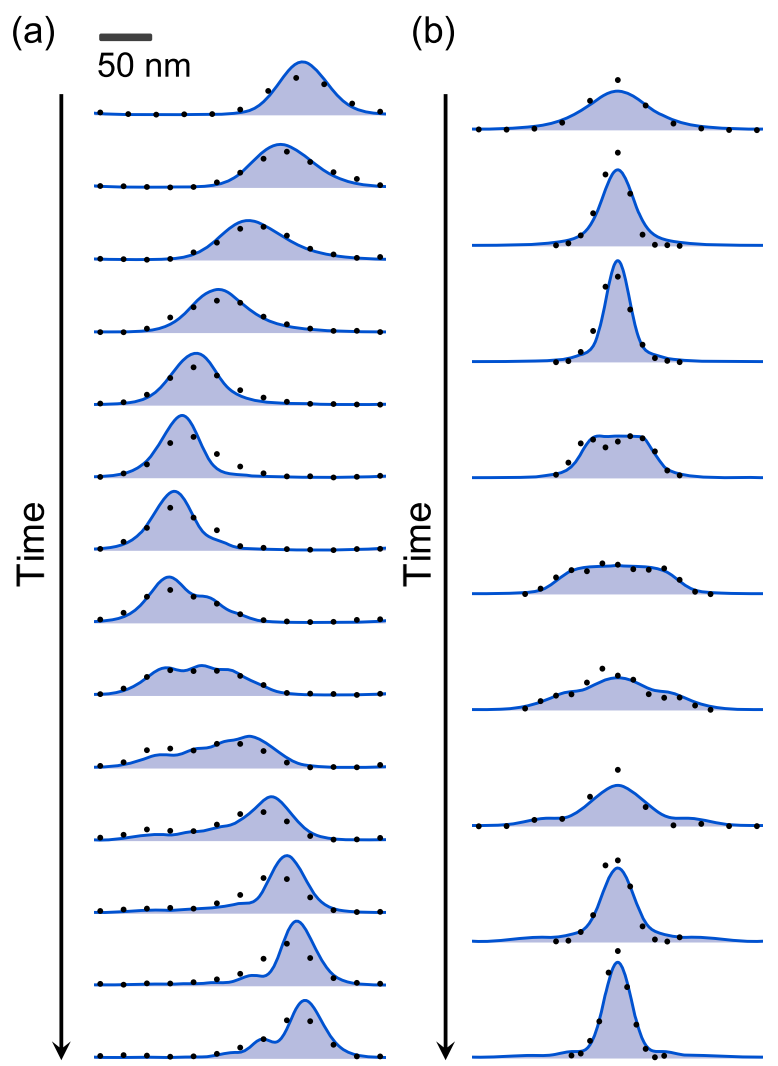

FIG. 3. Wave-function dynamics within the unit cell of an optical lattice. We excite the (a) sloshing motion and (b) breathing motion of $|\psi(x)|^{2}$ appears to have a wrong scale in a $140 E_{R}$-deep sinusoidal lattice by suddenly changing either the position or the depth of the lattice potential. $n(x)$ is plotted at different hold times [1 to $14 \mu \mathrm{s}$ in steps of $1 \mu \mathrm{s}$ for (a) and 1.5 to $9.5 \mu \mathrm{s}$ in steps of $1 \mu \mathrm{s}$ for (b)] after the sudden change. The points are experimental data with $\epsilon=0.05$, and the blue curves represent calculations of $n(x)$ based on the independently measured lattice parameters. Typical number uncertainties are $5 \%$ due to fluctuations from shot to shot.

$w_{0}=26.2_{-0.6}^{+1.6} \mathrm{~nm}$, where the uncertainty arises from the uncertainty in the Rabi-frequency calibrations used to determine the lattice depth. We measure $n(x)$ at this time with different resolution by varying $\epsilon$, as shown in Fig. 4. The measured width $w$ of $n(x)$ is plotted as the gray open circles, which decrease and approach the expected value for small $\epsilon$. Following Eq. (3), by deconvolving the results $[n(x)]$ with the calculated wave function $\left(|\psi(x)|^{2}\right)$ and taking into account the 800-ns expansion time, we estimate the intrinsic resolution $\sigma$ for different $\epsilon$, which is plotted as the black closed circles. The blue solid line is the calculated width of $f(x)$. The ultimate resolution is possibly limited by mechanical effects arising from the sharp potential associated with the dark state $[20,22,23]$. As the slice width $\sigma$ decreases, the total population in $\left|g_{2}\right\rangle$ also decreases, setting a practical limit on the usable resolution, as illustrated by the wave-function measurements shown in the Fig. 4 upper panel. The smallest measured $\sigma$ reaches $11.4_{-4.4}^{+2.0} \mathrm{~nm}$,

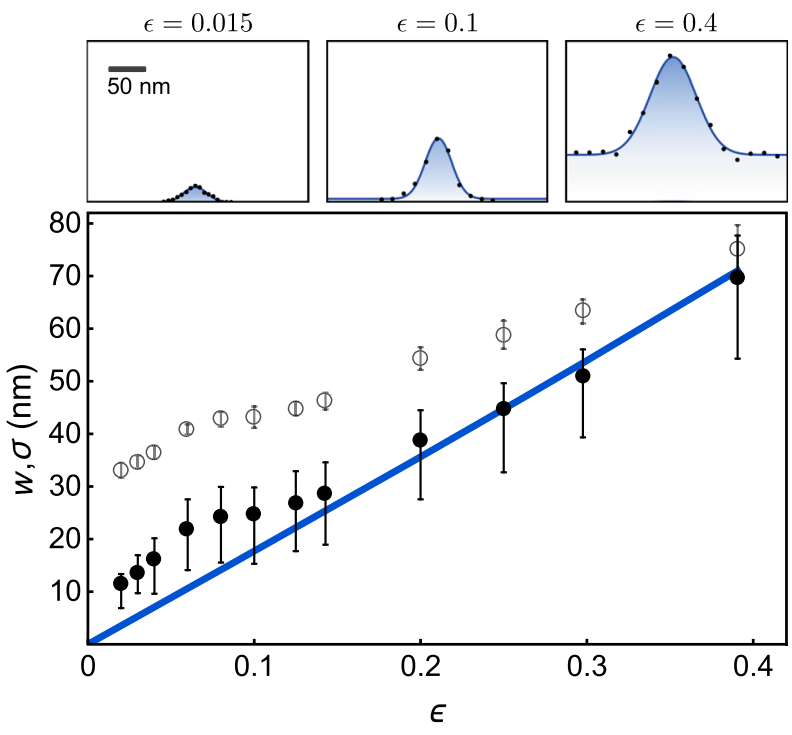

FIG. 4. Spatial resolution of the microscope. We create a narrow wave function $|\psi(x)|^{2}$ (FWHM $26 \mathrm{~nm}$ ) by exciting the breathing motion of atoms in a deep sinusoidal lattice and measure $n(x)$ at the focus point [see Fig. 3(b)] as a function of $\epsilon$. The measured $w$ from a Gaussian fit with a vertical offset to the $n(x)$ (see upper panel for typical wave-function measurements) is plotted against $\epsilon$ as the gray open circles, with the error bars showing 1 standard deviation from the fitting. These data are then deconvolved with the calculated wave function $|\psi(x)|^{2}$ to find the intrinsic resolution $\sigma$ and plotted as the black closed circles. The error bars are dominated by the systematic uncertainties in the width of $|\psi(x)|^{2}$. The blue curve is the calculated width of $f(x)$ at different $\epsilon$.

which could be improved with higher signal-to-noise ratio and Rabi frequencies.

\section{CONCLUSION}

We demonstrate superresolution imaging of atomic wave-function probability density with a spatial resolution of $\lambda / 50$ and a temporal resolution of $500 \mathrm{~ns}$. This imaging technique (demonstrated here on an ensemble of atoms) can be extended to single atoms by averaging over multiple realizations. The dark-state-based technique can be applied to image any atomic or molecular system as long as they host a three-level system, including the alkali atoms that are used in many experiments. Such high spatial and temporal resolution microscopy provides a new tool to address ultracold atom simulations of condensed-matter systems, especially phenomena associated with higher momenta and energies. For instance, the temporal resolution will allow us to measure the rapid dynamic evolution of the wave functions in periodically driven Floquet systems [24]. The spatial resolution of the technique could allow one to probe density fluctuations due to phonon and vortex excitations in a nonlattice cold atom system, which can be the size of the healing length (typically submicron) $[25,26]$. It is also useful 
in probing lattice systems with higher band population either intentionally populated to exploit the orbital d.o.f. [27,28] or due to band mixing from strong interactions [29]. As demonstrated in Fig. 3, our subwavelength resolution already allows us to distinguish the different atomic wave functions trapped in lattices with different subwavelength structure. Such a resolution will be critical in the study of optical lattices with lattice constants $\lambda / 2 N$ created through stroboscopic techniques [30], which is advantageous for studying many-body physics since the energy scale is $N^{2}$ times larger than a typical $\lambda / 2$ lattice. Finally, while the imaging technique demonstrated here measures the wavefunction probability density, the coherence of the dark-state selection process could allow for measurement of the local wave-function phase as well.

\section{ACKNOWLEDGMENTS}

We thank Victor M. Galitski, Alexey V. Gorshkov, and Przemyslaw Bienias for fruitful discussions. We also want to thank Carlos Bracamontes Palma for DDS/FPGA hardware development. This work is supported by NSF Physics Frontier Center, PFC@JQI (PHY1430094) and ONR (Grant No. N000141712411).

S. S. and Y. W. contributed equally to this work.

Note added.-Recently, we became aware of similar work from Cheng Chin's group [34].

\section{APPENDIX A: ${ }^{171}$ YB ATOM LEVEL STRUCTURE}

We measure the $\mathrm{Yb}$ atoms by absorption imaging on the ${ }^{1} S_{0}-{ }^{1} P_{1}$ transition with light at $399 \mathrm{~nm}$ generated by a frequency-doubled laser system. We stabilize the seed of the imaging laser (at $800 \mathrm{~nm}$ ) via a scanning transfer cavity lock $[31,32]$ with the master laser locked to a saturated absorption feature on the ${ }^{85} \mathrm{Rb}\left|5^{2} S_{1 / 2}, F=3\right\rangle \leftrightarrow\left|5^{2} P_{3 / 2}, F^{\prime}=3-4\right\rangle$ crossover signal. State selectivity is achieved by imaging in a large magnetic field of $36 \mathrm{mT}$ along $\hat{x}$, such that the resulting $440-\mathrm{MHz}$ separation between the $|6\rangle$ and $|7\rangle$ Zeeman sublevels of ${ }^{1} P_{1}$ is much larger than the linewidth $\Gamma_{{ }^{1}} P_{1}=$ $2 \pi \times 27.9 \mathrm{MHz}$. The imaging beam propagates along $\hat{x}$ with $\sigma^{+}$polarization relative to $\vec{B}$. We measure the population in the $\left|g_{2}\right\rangle$ hyperfine ground state by making the laser resonant with its respective stretched state, $\left|g_{2}\right\rangle \leftrightarrow|6\rangle$ for $10 \mu \mathrm{s}$. Optical pumping of atoms in $\left|g_{1}\right\rangle$ into $\left|g_{2}\right\rangle$ via $|7\rangle$ is suppressed by a factor of over 3000 due to the $440-\mathrm{MHz}$ detuning and the short imaging time.

Figure 5 depicts the three hyperfine states that constitute the $\Lambda$ system consisting of $\left|g_{1}\right\rangle,\left|g_{2}\right\rangle$, and $|e\rangle$ that we use to generate the KP lattice and to probe the wave function of arbitrary lattices. We create the off-resonant sinusoidal acStark-shift lattices using the $\left|g_{1}\right\rangle \leftrightarrow|5\rangle$ transition with the lattice depth given by $3 \Omega_{c}^{2}(x) / 8 \Delta_{\mathrm{HFS}}$. The effect of this offresonant lattice is negligible when the atoms are in the KP

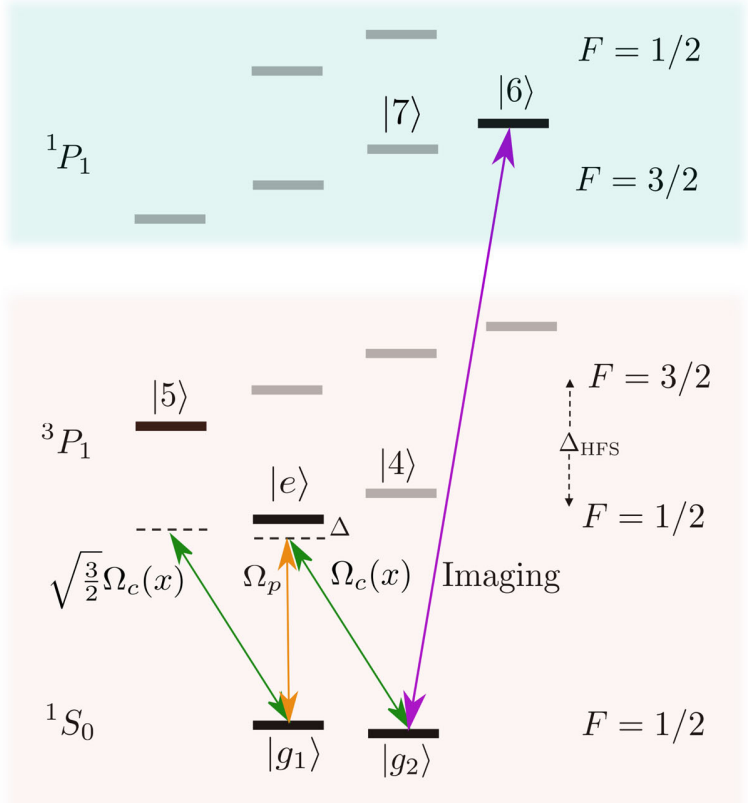

FIG. 5. Level structure of the ${ }^{1} S_{0},{ }^{3} P_{1}$, and ${ }^{1} P_{1}$ manifolds of ${ }^{171} \mathrm{Yb}: \Delta$ is the single-photon detuning and $\Delta_{\mathrm{HFS}} \sim 6 \mathrm{GHz}$ is the ${ }^{3} P_{1}$ hyperfine splitting.

lattice with $\Omega_{c}=70 \Gamma$ and $\Omega_{p}=10 \Gamma$, where $\Gamma=2 \pi \times$ $182 \mathrm{kHz}$ is the inverse lifetime of ${ }^{3} P_{1}$. The method we use to calibrate our Rabi frequencies is detailed in Ref. [20].

\section{APPENDIX B: EXPERIMENTAL SEQUENCE}

Preparation and experimental sequence: Before the start of each experimental sequence, the atoms are optically pumped into $\left|g_{1}\right\rangle$.

Stage I: To simplify the study of the static and dynamics properties of wave functions in lattices, we prepare our atom cloud to fill only the ground band of the lattice of interest. Since the Fermi energy of our atomic cloud is approximately $3 E_{R}$, adiabatic loading into the lattice will have some population in the first excited band. We resolve this issue by first loading atoms into a KP lattice with $\epsilon=$ $0.14\left(\Omega_{c 1}=\Omega_{c 2}=35 \Gamma, \Omega_{p}=10 \Gamma\right.$, and $\left.\Delta=4 \mathrm{MHz}\right)$ [20] and then holding for $5 \mathrm{~ms}$. Atoms in higher bands of the KP lattice have a shorter lifetime and are lost from the trap, effectively removing atoms in the higher bands.

Stage II: In this stage, we adiabatically transfer atoms from the ground band of the KP lattice into the ground band of an ac-Stark-shift lattice in $10 \mathrm{~ms} . \Delta$ is ramped down to 0 , which is important in achieving the maximum speed while adiabatically following the dynamic dark state in stage IV.

Stage III: In this stage, we excite dynamics in the lattice. In Fig. 6(c), the phase of the $\Omega_{c 1}$ beam is ramped to $90^{\circ}$ in $100 \mathrm{~ns}$, so as to diabatically shift the position of the lattice by $\lambda / 8$ which induces sloshing dynamics. In Fig. $6(d)$, the lattice depth is suddenly increased from $6 E_{R}$ to $140 E_{R}$, 
and the atoms are held in the deep lattice for different times (1.5 to $9.5 \mu \mathrm{s}$ ) to study the breathing motion.

Stage IV: In this stage, we measure the wave function. First, $\Omega_{c 2}$ is suddenly turned off to 0 , while $\Omega_{c 1}$ is set to $250 \Gamma$. Then, the $\Omega_{p}$ beam is suddenly turned on to its desired value. Because of the large energy separation of $125 \Gamma\left(\Omega_{c 1}=250 \Gamma\right.$ and $\left.\Omega_{p}=\Omega_{c 2}=0\right)$ between the dark and bright states, the adiabatic following of the dark state is guaranteed during the turn-on of the $\Omega_{p}$ beam. Then $\Omega_{c 2}$ beam is turned on with a different phase $\phi_{c 2}$ implemented by changing the phase of the rf drive to the acousto-optic modulator (AOM), with the amplitude being ramped up to $250 \Gamma$ with the optimal waveform so as to preserve the adiabaticity during the ramp. By scanning $\phi_{c 2}$ from $0^{\circ}$ to $360^{\circ}$, we change the position of the node of $\Omega_{c}(x)=500 \Gamma \cos (k x)$, thereby mapping out the probability amplitude of atoms in each spatial slice of the wave function.

Stage V: Finally, the lattice beams are ramped off simultaneously in $100 \mathrm{~ns}$ by switching off the rf drive to the AOMs. Since the dark-state composition depends only on the ratio $\left(\Omega_{c 1}+\Omega_{c 2}\right) / \Omega_{p}$ and not on the absolute magnitude of the Rabi frequencies, simultaneous ramp-off of the lattice beams preserves the dark-state composition until the atoms are imaged.

\section{APPENDIX C: HARDWARE CONTROL}

In order to generate the experimental sequences described earlier, we need to have fine, high-bandwidth control over the amplitude and phase of the light fields $\Omega_{c 1}, \Omega_{c 2}$, and $\Omega_{p}$. This control is achieved by using a home-built fieldprogrammable-gate-array- (FPGA; Spartan 6) controlled direct-digital-synthesis- (DDS; AD9910) based rf signal generator. We use three such devices to drive three AOMs for the light fields. Phase coherence between the light fields is ensured by having the devices be clocked by the same $10-\mathrm{MHz}$ clock source and having the light fields be derived from the same laser. Each device generates an $80-\mathrm{MHz}$ rf carrier signal with arbitrary amplitude and phase and imprints that onto the light via its respective AOM. The DDS can update the phase of the rf signal every $4 \mathrm{~ns}$. The desired amplitude waveform (AW) is loaded into the local RAM of the FPGA of the device and is updated at a maximum update rate of $8 \mathrm{~ns}$. The maximum length of the AW pulse is approximately $256 \mu$ s when updated every $8 \mathrm{~ns}$.
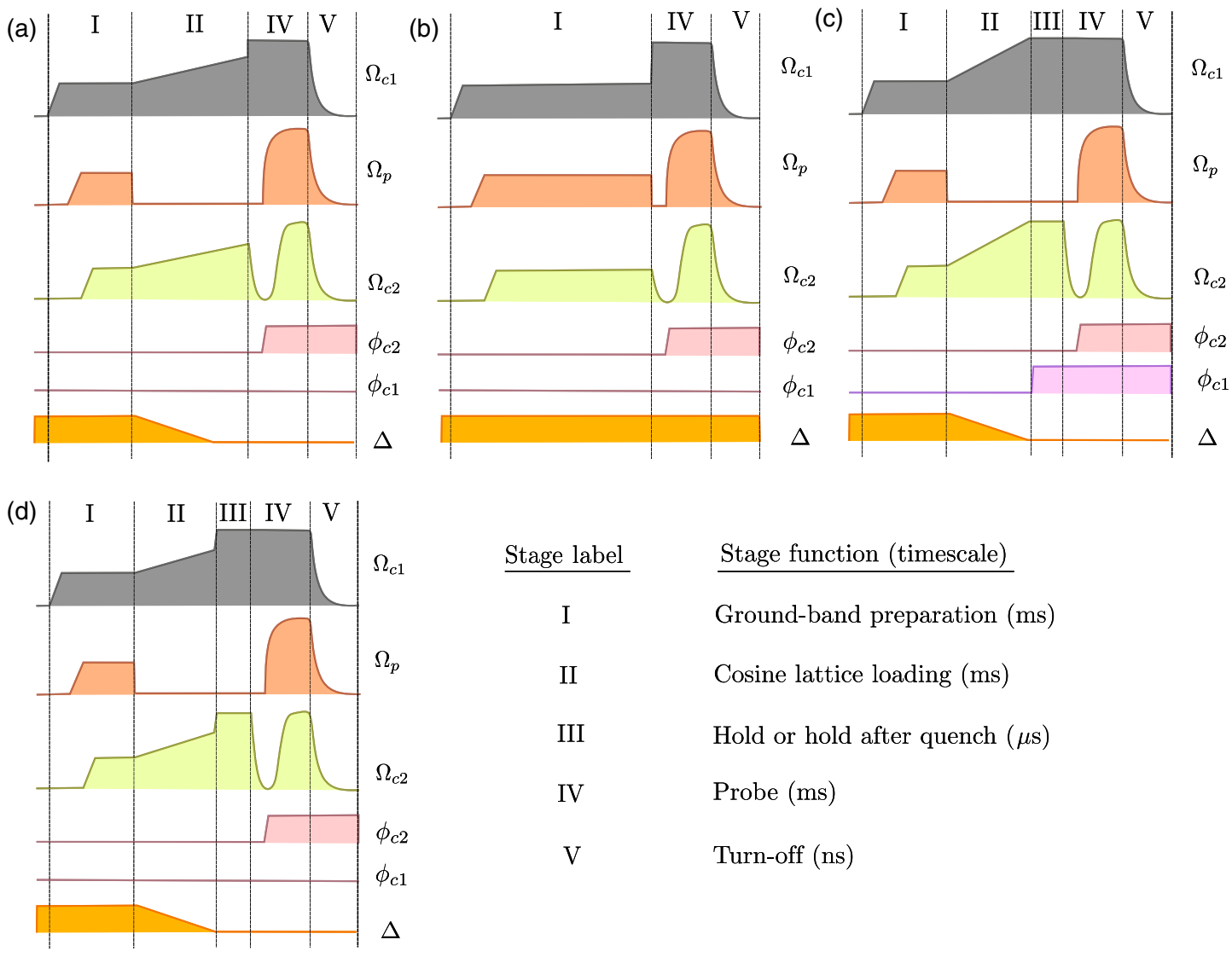

FIG. 6. Experimental sequences. (a) Probing the ground-state wave function of a sinusoidal lattice. (b) Probing the ground-state wave function of a KP lattice. (c) Probing the dynamics after a sudden change in the lattice position. (d) Probing the dynamics after a sudden change in lattice depth. The drawings are not to scale. 


\section{APPENDIX D: OPTIMAL AMPLITUDE WAVEFORM FOR STIRAP}

During stage IV, we adiabatically transfer atoms from $|1\rangle$ to $|2\rangle$ near the node of $\Omega_{c}(x)$ via STIRAP [19]. For an ideal $\Lambda$ system, the local adiabatic criterion is given by Ref. [19] as $\Omega_{\mathrm{rms}} \gg\left|\Omega_{c} \dot{\Omega}_{p}-\dot{\Omega}_{c} \Omega_{p}\right| / \Omega_{\mathrm{rms}}^{2}$, where $\Omega_{\mathrm{rms}}=\sqrt{\Omega_{c}^{2}+\Omega_{p}^{2}}$ (at $\Delta=0$ ) is the energy gap between the dark and bright eigenstates and the rhs is the offdiagonal coupling between them. We define an adiabaticity parameter $r$,

$$
\Omega_{\mathrm{rms}}=r \frac{\left|\Omega_{c} \dot{\Omega}_{p}-\dot{\Omega}_{c} \Omega_{p}\right|}{\Omega_{\mathrm{rms}}^{2}} .
$$

A larger value of $r$ implies a more adiabatic but slower transfer. The equation is solved to give an optimal shape of $\Omega_{c 2}$ near the node of $\Omega_{c}(x)\left(\Omega_{c 1}\right.$ and $\Omega_{p}$ are kept constant here) for stage IV:

$$
\Omega_{c 2}(t)=\Omega_{c 1}-\Omega_{p} \frac{\left(\frac{\Omega_{c 1}}{\sqrt{\Omega_{c 1}^{2}+\Omega_{p}^{2}}}-\frac{\Omega_{p} t}{r}\right)}{\sqrt{1-\left(\frac{\Omega_{c 1}}{\sqrt{\Omega_{c 1}^{2}+\Omega_{p}^{2}}}-\frac{\Omega_{p} t}{r}\right)^{2}}} .
$$

The time it takes to finish the $\Omega_{c 2}(t)$ ramp is

$$
T_{r}=\frac{r}{\Omega_{p}} \frac{1}{\sqrt{1+4 \epsilon^{2}}} .
$$

For a typical value of $r=15, \Omega_{c 1}=250 \Gamma$, and $\Omega_{p}=$ $25 \Gamma, T_{r}$ is $0.52 \mu \mathrm{s}$; see Fig. 7. For a given spatial resolution determined by $\epsilon$, more available laser power will reduce $T_{r}$ and increase the temporal resolution.

We experimentally investigate the minimum $r$ required to ensure adiabatic following of the dynamic dark state. We do this by keeping $r$ fixed and measuring the temperature of the cloud after STIRAP pulses. If the adiabaticity is not well satisfied, the nonzero probability of atoms being in the excited state $|e\rangle$ leads to scattering which increases the temperature of the atoms. To increase the sensitivity of the measurement, we apply ten successive STIRAP pulses. This study is performed with only one control beam $\Omega_{c 2}$

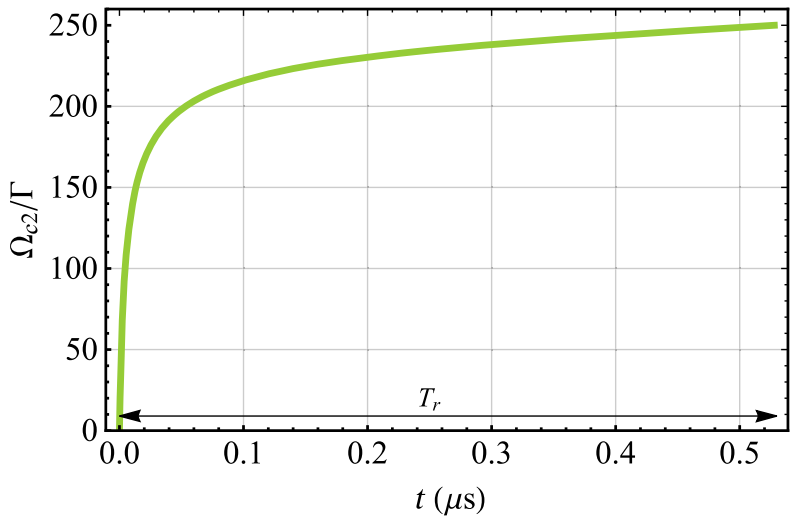

FIG. 7. The optimal amplitude waveform for $\Omega_{c 2}(t)$ for $\Omega_{c 1}=250 \Gamma, \Omega_{p}=25 \Gamma$.
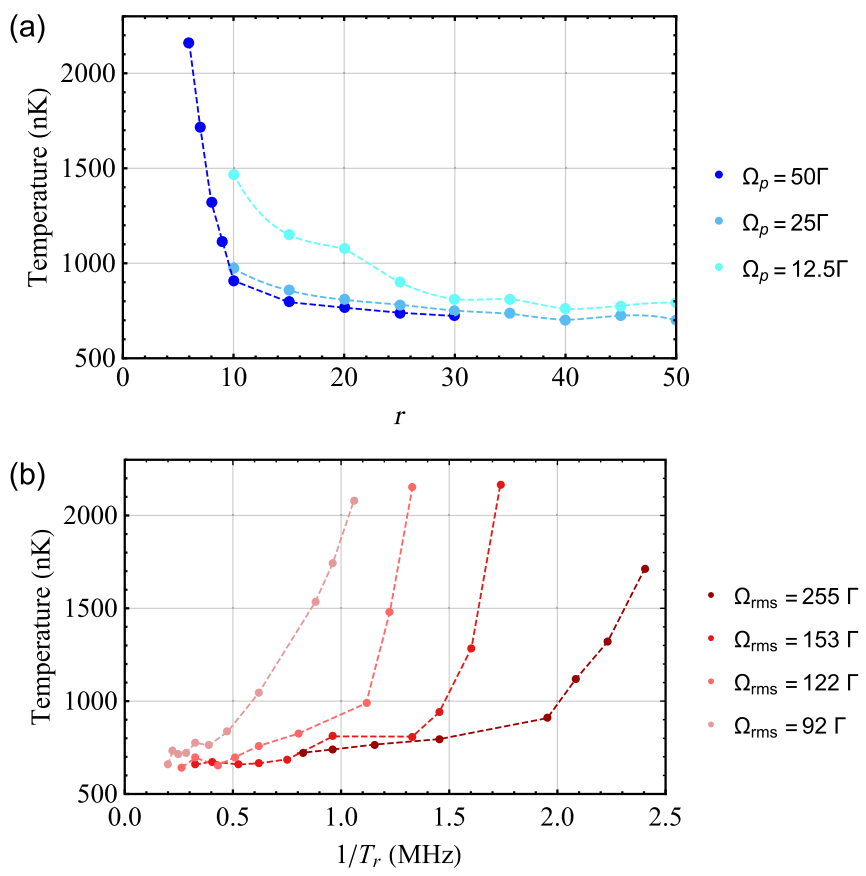

FIG. 8. (a) The temperature of the atoms after ten complete STIRAP pulses for different values of $r$ and $\Omega_{p}$ and (b) different values of $\Omega_{\mathrm{rms}}$ and $T_{r}$

and the probe beam $\Omega_{p}$. In each pulse, $\Omega_{c 2}$ is ramped up from 0 to $250 \Gamma$ and then ramped down to 0 following the optimal waveform described by Eq. (D1). After ten cycles, the temperature of the cloud is measured, and the results are shown in Fig. 8(a). One can see that beyond a certain value of $r$, the STIRAP process becomes adiabatic; i.e., the temperature is independent of $r$, which occurs at about $r=15$ for $\Omega_{p}=50 \Gamma, 25 \Gamma$. Below $r=15$, the local adiabaticity criterion breaks down. During the probe stage in Fig. 6, we use $r=15$ in Eq. (D2) to calculate the optimal AWs.

The energy gap between the dark and bright eigenstates increases with increasing $\Omega_{\text {rms }}$, which reduces $T_{r}$ needed to ensure adiabaticity. We study this effect by keeping the ratio of $\Omega_{p} / \Omega_{c 2}=0.2$ constant while $\Omega_{\mathrm{rms}}$ is varied. As shown in Fig. 8(b), with larger $\Omega_{\mathrm{rms}}$, faster ramp speed can be achieved while still being adiabatic.

\section{APPENDIX E: PRESERVING THE DARK-STATE COMPOSITION DURING RAMP-OFF}

The ramp-off stage of the lattice beams is crucial for our measurement since it must preserve the dark-state composition generated during the probing stage. We achieve this requirement by ramping down the light fields simultaneously while maintaining a fixed ratio between the Rabi frequencies $\Omega_{c i}(t) / \Omega_{p}(t)$, where $i=1,2$. The dark-state composition is thus preserved, as it depends only on the ratio and not on the absolute magnitudes of the Rabi frequencies. For the typical Rabi frequencies we use in the experiments, the relative delay between the light fields 
needs to be less than $20 \mathrm{~ns}$ to preserve the dark-state composition. The ramp-off must be diabatic with respect to the mechanical d.o.f. of the wave function. This is guaranteed by turning off the rf drive to the AOMs simultaneously in $100 \mathrm{~ns}$.

Experimentally, simultaneous turn-off of the light fields between different AOMs is not guaranteed, as delays may exist due to the laser light hitting the AOM crystals at different distances from their respective piezoelectric transducers. With our best alignment of the AOMs, we reduce the delay of the light fields to within $60 \mathrm{~ns}$ of each other. This remnant delay at the atoms is compensated by delaying the digital trigger that turns off the rf drive to an AOM. We accurately measure the turn-off delays between the light fields at the atoms using the method shown in Fig. 9(a). Sweeping $t_{\text {shift }}$ of the digital trigger, thereby changing $\Delta t=$ $t_{\text {shift }}-t_{\text {delay }}$, we measure the atom number in $\left|g_{2}\right\rangle$ using state-selective imaging. We are able to change $t_{\text {shift }}$ at picosecond timescales using a delayed-pulse generator (SRS DG535). When $\Delta t<0$, the spin composition of the dark-state wave function is $\left|g_{1}\right\rangle$. But as $\Delta t \geq 0$, the dark-state composition starts to become predominantly $\left|g_{2}\right\rangle$ with increase in $\Delta t$ as shown in Fig. 8(b). By fitting a line to the data, we get the time at which the dark-state spin composition just starts to change from $\left|g_{1}\right\rangle$ to $\left|g_{2}\right\rangle$. The

(a)

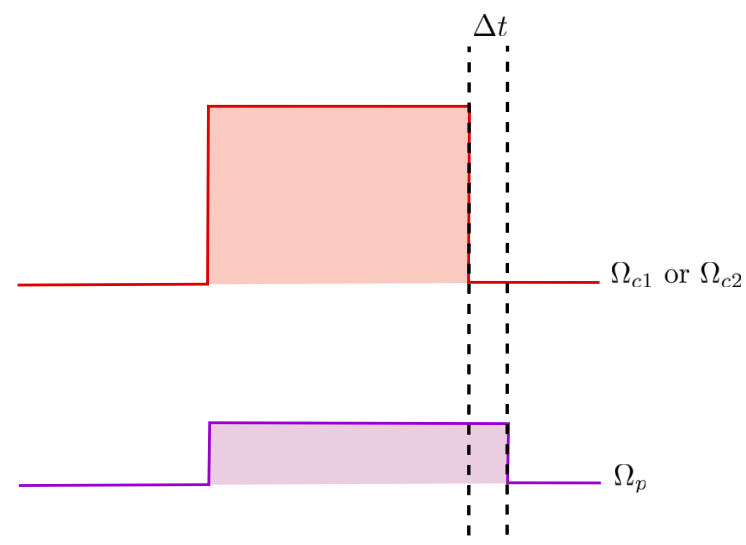

(b)

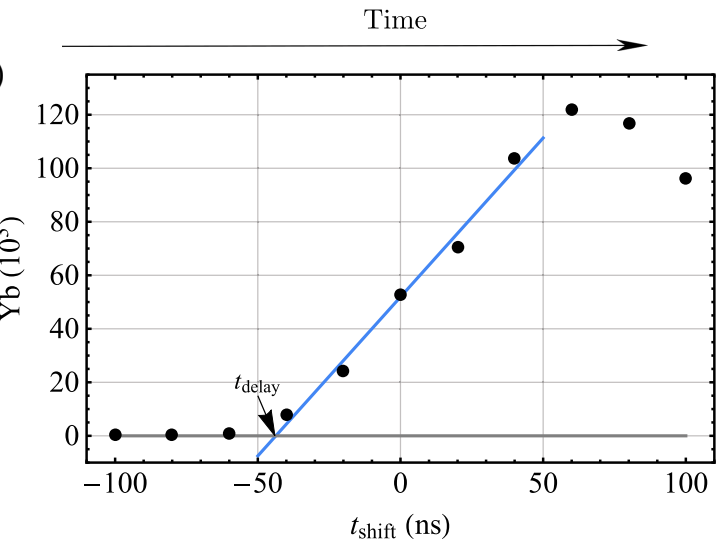

FIG. 9. (a) Optical pulse sequence used to optimize the temporal overlap between the $\Omega_{c i}$ and $\Omega_{p}$ light fields. (b) The population of the atoms in the $\left|g_{2}\right\rangle$ state as a function of $t_{\text {shift }}$ with $\Omega_{c 1}=250 \Gamma$ and $\Omega_{p}=50 \Gamma$. $\left|g_{2}\right\rangle$ component of the dark state is close to 0 for the Rabi frequencies used in the measurement when $\Delta t=0$. By measuring these delays for each pair of beams $\Omega_{c 1}$ and $\Omega_{p}$, $\Omega_{c 2}$ and $\Omega_{p}$, we can compensate them via adjusting the lengths of the BNC cables of the digital trigger to the rf sources.

Another approach to ensure that the dark-state composition does not change is to turn off the light fields diabatically with respect to the spin d.o.f. of the dark-state wave function. As the Rabi frequencies of the light fields are in the range of hundreds of megahertz, the turn-off time must be less than $10 \mathrm{~ns}$. It is challenging to achieve such turn-off times with AOMs, but one could use electro-optical modulators instead.

\section{APPENDIX F: THEORY AND CALCULATION}

The eigenfunctions of atoms in an optical lattice are given by the Bloch ansatz as $\phi_{q}(x)=e^{i q x} u_{q}(x)$, where $u_{q}(x)=u_{q}(x+a), q \in[-k, k]$ is the quasimomentum, and $a$ is the periodicity of the lattice. The field operator for a spin $|\sigma\rangle, \Psi_{\sigma}^{\dagger}(x)$, and the total field operator for a spin- $1 / 2$ particle in a lattice $\Psi_{S}^{\dagger}(x)$ is given as [33]

$$
\begin{aligned}
& \Psi_{\sigma}^{\dagger}(x)=\sum_{q=-k}^{q=k} \phi_{q}^{*}(x) c_{q \sigma}^{\dagger}, \\
& \Psi_{S}^{\dagger}(x)=\left[\Psi_{\left|g_{1}\right\rangle}^{\dagger}(x), \Psi_{\left|g_{2}\right\rangle}^{\dagger}(x)\right]^{T},
\end{aligned}
$$

where $\left\{c_{q \sigma}, c_{q^{\prime} \sigma^{\prime}}^{\dagger}\right\}=\delta_{q q^{\prime}} \delta_{\sigma \sigma^{\prime}}$.

Before stage IV, all atoms are in $\left|g_{1}\right\rangle$ as the trivial dark state which is represented by the total field operator $\Psi_{S}^{\dagger}(x)=\Psi_{\left|g_{1}\right\rangle}^{\dagger}(x)(1,0)^{T}$. During stage IV, the adiabatic preparation of the dark-state wave function is given as

$\Psi_{S}^{\dagger}(x)=\Psi_{\left|g_{1}\right\rangle}^{\dagger}(x)\left(\frac{s \sin (k x)}{\sqrt{s^{2} \sin ^{2}(k x)+1}}, \frac{1}{\sqrt{s^{2} \sin ^{2}(k x)+1}}\right)^{T}$,

where $s=\frac{1}{\epsilon}=\left(\Omega_{c 1}+\Omega_{c 2}\right) / \Omega_{p}$.

The measurement involves probing the probability density of atoms in $\left|g_{2}\right\rangle$ averaged over the filled ground band of the optical lattice $(|\mathrm{GB}\rangle)$ using state-selective imaging. The observable that we measure is therefore,

$$
\begin{aligned}
& \left\langle g_{2}\left|\left\langle\mathrm{~GB}\left|\Psi_{S}^{\dagger}(x) \Psi_{S}(x)\right| \mathrm{GB}\right\rangle\right| g_{2}\right\rangle \\
& =\left(\frac{1}{s^{2} \sin ^{2}(k x)+1}\right) \sum_{q=-k}^{q=k} \sum_{q^{\prime}=-k}^{q^{\prime}=k} \phi_{q}^{*}(x) \phi_{q^{\prime}}(x) \\
& \left.\quad \times\left\langle\mathrm{GB} \mid c_{q\left|g_{1}\right\rangle}^{\dagger} c_{q^{\prime}\left|g_{1}\right\rangle}\right\rangle \mathrm{GB}\right\rangle \\
& =\left(\frac{1}{s^{2} \sin ^{2}(k x)+1}\right) \sum_{q=-k}^{q=k}\left|\phi_{q}(x)\right|^{2} \\
& =f(x) \sum_{q=-k}^{q=k}\left|\phi_{q}(x)\right|^{2} .
\end{aligned}
$$


Therefore, the measured density distribution within a unit cell is the convolution of the actual density distribution $\sum_{q=-k}^{q=k}\left|\phi_{q}(x)\right|^{2}$ and the probing function $f(x)$.

We solve for the band structure of two types of lattices: the KP lattice and the sinusoidal ac-Stark-shift lattice. Using the Bloch ansatz, the Schrödinger equation can be written as

$$
\left(\frac{\hbar^{2}}{2 m}\left(-i \hbar \partial_{x}+q\right)^{2}+V(x)\right) u_{q}(x)=\epsilon(q) u_{q}(x) .
$$

The Schrödinger equation can be solved numerically by Fourier expansion of $u_{q}(x)$ into plane waves

$$
u_{q}(x)=\sum_{n=-N}^{N} c_{n, q} e^{i n k x},
$$

where $n \in(0,1,2, \ldots)$ is the band index and diagonalizing the matrix equation resulting in this basis. Similarly, the time dependence of the wave function after suddenly changing the lattice can be calculated by solving the time-dependent Schrödinger equation with appropriate initial conditions.

[1] G. Binnig and H. Rohrer, Scanning Tunneling Microscopy, Surf. Sci. 126, 236 (1983).

[2] S. W. Hell, Far-Field Optical Nanoscopy, Science 316, 1153 (2007).

[3] C. Gross and I. Bloch, Quantum Simulations with Ultracold Atoms in Optical Lattices, Science 357, 995 (2017).

[4] G. S. Agarwal and K. T. Kapale, Subwavelength Atom Localization via Coherent Population Trapping, J. Phys. B 39, 3437 (2006).

[5] J. A. Miles, Z. J. Simmons, and D. D. Yavuz, Subwavelength Localization of Atomic Excitation Using Electromagnetically Induced Transparency, Phys. Rev. X 3, 031014 (2013).

[6] T. Gericke, P. Würtz, D. Reitz, T. Langen, and H. Ott, HighResolution Scanning Electron Microscopy of an Ultracold Quantum Gas, Nat. Phys. 4, 949 (2008).

[7] A. Alberti, C. Robens, W. Alt, S. Brakhane, M. Karski, R. Reimann, A. Widera, and D. Meschede, Super-Resolution Microscopy of Single Atoms in Optical Lattices, New J. Phys. 18, 053010 (2016).

[8] K. D. Nelson, X. Li, and D. S. Weiss, Imaging Single Atoms in a Three-Dimensional Array, Nat. Phys. 3, 556 (2007).

[9] A. V. Gorshkov, L. Jiang, M. Greiner, P. Zoller, and M. D. Lukin, Coherent Quantum Optical Control with Subwavelength Resolution, Phys. Rev. Lett. 100, 093005 (2008).

[10] E. Paspalakis and P. L. Knight, Localizing an Atom via Quantum Interference, Phys. Rev. A 63, 065802 (2001).

[11] J.-T. Chang, J. Evers, M. O. Scully, and M. S. Zubairy, Measurement of the Separation between Atoms beyond Diffraction Limit, Phys. Rev. A 73, 031803 (2006).
[12] J. Cho, Addressing Individual Atoms in Optical Lattices with Standing-Wave Driving Fields, Phys. Rev. Lett. 99, 020502 (2007).

[13] D. D. Yavuz and N. A. Proite, Nanoscale Resolution Fluorescence Microscopy Using Electromagnetically Induced Transparency, Phys. Rev. A 76, 041802 (2007).

[14] H. Li, V. A. Sautenkov, M. M. Kash, A. V. Sokolov, G. R. Welch, Y. V. Rostovtsev, M. S. Zubairy, and M. O. Scully, Optical Imaging beyond the Diffraction Limit via Dark States, Phys. Rev. A 78, 013803 (2008).

[15] Y. Ashida and M. Ueda, Diffraction-Unlimited Position Measurement of Ultracold Atoms in an Optical Lattice, Phys. Rev. Lett. 115, 095301 (2015).

[16] D. Yang, C. Laflamme, D. V. Vasilyev, M. A. Baranov, and P. Zoller, Theory of a Quantum Scanning Microscope for Cold Atoms, Phys. Rev. Lett. 120, 133601 (2018).

[17] P. C. Maurer, J. R. Maze, P. L. Stanwix, L. Jiang, A. V. Gorshkov, A. A. Zibrov, B. Harke, J. S. Hodges, A. S. Zibrov, A. Yacoby, D. Twitchen, S. W. Hell, R. L. Walsworth, and M. D. Lukin, Far-Field Optical Imaging and Manipulation of Individual Spins with Nanoscale Resolution, Nat. Phys. 6, 912 (2010).

[18] D. Yang, D. V. Vasilyev, C. Laflamme, M. A. Baranov, and P. Zoller, Quantum Scanning Microscope for Cold Atoms, Phys. Rev. A 98, 023852 (2018).

[19] N. V. Vitanov, A. A. Rangelov, B. W. Shore, and K. Bergmann, Stimulated Raman Adiabatic Passage in Physics, Chemistry, and Beyond, Rev. Mod. Phys. 89, 015006 (2017).

[20] Y. Wang, S. Subhankar, P. Bienias, and M. Łącki, T.-C. Tsui, M. A. Baranov, A. V. Gorshkov, P. Zoller, J. V. Porto, and S. L. Rolston, Dark State Optical Lattice with a Subwavelength Spatial Structure, Phys. Rev. Lett. 120, 083601 (2018).

[21] V. D. Vaidya, J. Tiamsuphat, S. L. Rolston, and J. V. Porto, Degenerate Bose-Fermi Mixtures of Rubidium and Ytterbium, Phys. Rev. A 92, 043604 (2015).

[22] F. Jendrzejewski, S. Eckel, T. G. Tiecke, and G. Juzeliūnas, G. K. Campbell, Liang Jiang, and A. V. Gorshkov, Subwavelength-Width Optical Tunnel Junctions for Ultracold Atoms, Phys. Rev. A 94, 063422 (2016).

[23] M. Łącki, M. A. Baranov, H. Pichler, and P. Zoller, Nanoscale "Dark State” Optical Potentials for Cold Atoms, Phys. Rev. Lett. 117, 233001 (2016).

[24] N. Goldman and J. Dalibard, Periodically Driven Quantum Systems: Effective Hamiltonians and Engineered Gauge Fields, Phys. Rev. X 4, 031027 (2014).

[25] K. W. Madison, F. Chevy, W. Wohlleben, and J. Dalibard, Vortex Formation in a Stirred Bose-Einstein Condensate, Phys. Rev. Lett. 84, 806 (2000).

[26] S. Krönke, M. Pyzh, C. Weitenberg, and P. Schmelcher, Quantum Point Spread Function for Imaging Trapped Few-Body Systems with a Quantum Gas Microscope, arXiv:1806.08982.

[27] G. Wirth, M. Ölschläger, and A. Hemmerich, Evidence for Orbital Superfluidity in the p-Band of a Bipartite Optical Square Lattice, Nat. Phys. 7, 147 (2011).

[28] X. Li and W. V. Liu, Physics of Higher Orbital Bands in Optical Lattices: A Review, Rep. Prog. Phys. 79, 116401 (2016). 
[29] A. Goban, R. B. Hutson, G. E. Marti, S. L. Campbell, M. A. Perlin, P. S. Julienne, J. P. D'Incao, A. M. Rey, and J. Ye, Emergence of Multi-Body Interactions in a Fermionic Lattice Clock, Nature (London) 563, 369 (2018).

[30] S. Nascimbene, N. Goldman, N. R. Cooper, and J. Dalibard, Dynamic Optical Lattices of Subwavelength Spacing for Ultracold Atoms, Phys. Rev. Lett. 115, 140401 (2015).

[31] S. Subhankar, A. Restelli, Y. Wang, S. L. Rolston, and J. V. Porto, Microcontroller Based Scanning Transfer Cavity Lock for Long-Term Laser Frequency Stabilization, arXiv:1810.07256.
[32] J. H. T. Burke, O. Garcia, K. J. Hughes, B. Livedalen, and C. A. Sackett, Compact Implementation of a Scanning Transfer Cavity Lock, Rev. Sci. Instrum. 76, 116105 (2005).

[33] R. A. Jishi, Feynman Diagram Techniques in Condensed Matter Physics (Cambridge University Press, Cambridge, England, 2013).

[34] M. McDonald, J. Trisnadi, K.-X. Yao, and C. Chin, preceding paper, Super-Resolution Microscopy of Cold Atoms in an Optical Lattice, Phys. Rev. X 9021001 (2018). 